\title{
Delayed response of the global total electron content to solar EUV variations
}

\author{
Christoph Jacobi $^{1}$, Norbert Jakowski ${ }^{2}$, Gerhard Schmidtke ${ }^{3}$, and Thomas N. Woods ${ }^{4}$ \\ ${ }^{1}$ Leipzig Institute for Meteorology, Universität Leipzig, Stephanstr. 3, 04103 Leipzig, Germany \\ ${ }^{2}$ German Aerospace Center, Kalkhorstweg 53, 17235 Neustrelitz, Germany \\ ${ }^{3}$ Fraunhofer-Institut für Physikalische Messtechnik (IPM), Heidenhofstraße 8, 79110 Freiburg, Germany \\ ${ }^{4}$ LASP, University of Colorado, 3665 Discovery Dr., Boulder, CO 80303, USA
}

Correspondence to: Christoph Jacobi (jacobi@uni-leipzig.de)

Received: 27 November 2015 - Revised: 24 February 2016 - Accepted: 4 April 2016 - Published: 28 September 2016

\begin{abstract}
The ionospheric response to solar extreme ultraviolet (EUV) variability during 2011-2014 is shown by simple proxies based on Solar Dynamics Observatory/Extreme Ultraviolet Variability Experiment solar EUV spectra. The daily proxies are compared with global mean total electron content (TEC) computed from global TEC maps derived from Global Navigation Satellite System dual frequency measurements. They describe about $74 \%$ of the intra-seasonal TEC variability. At time scales of the solar rotation up to about 40 days there is a time lag between EUV and TEC variability of about one day, with a tendency to increase for longer time scales.
\end{abstract}

\section{Introduction}

The solar extreme ultraviolet (EUV) radiation varies on different time scales, including the 27-day Carrington rotation as one of the primary sources of variability at the intraseasonal time scale. Consequences are strong changes of the Total Electron Content (TEC), which is the vertically integrated electron density of the ionosphere/plasmasphere systems, often given in terms of TEC Units (TECU, 1 TECU = $10^{16}$ electrons $\mathrm{m}^{-2}$ ). The majority of the electrons are found in the ionospheric $\mathrm{F}$ layer where, according to simple theory based on an oxygen dominated thermosphere, the electron density is proportional to the ionization rate. Therefore, TEC variability is a coarse estimate for ionization as well, so that indices describing ionization may be compared against ionospheric TEC or, in turn, these indices may be used to provide a first estimate of ionospheric TEC. In addition to the frequently used F10.7 (solar radio flux at $10.7 \mathrm{~cm}$ ) index measured by the National Research Council of Canada (NRCC, 2014), proxies based on solar EUV, like E10.7 (Tobiska, 2001) are used to characterize the solar variability and its influence on the atmosphere/ionosphere. It has been found that other proxies based on EUV measurements or combination of proxies may characterize ionospheric variations better (e.g. Maruyama, 2010). Also using the MgII index has been found to be a better representation when used in atmospheric models or to describe the ionosphere (Thuillier and Bruinsma, 2001; Maruyama, 2010). Unglaub et al. (2011, 2012) have introduced a proxy, termed EUV-TEC, which is based on the vertically and globally integrated primary ionization rates. It has been calculated from spectral EUV fluxes measured by the Solar EUV Experiment (SEE) on board the Thermosphere Ionosphere Mesosphere Energetics and Dynamics (TIMED) satellite (Woods et al., 2000, 2005). Using data of about one decade, Unglaub et al. $(2011,2012)$ reconfirmed that ionization calculations based on the measured spectra describe the TEC variability better than e.g. F10.7.

Parameters describing ionospheric electron density and EUV proxies are not always in phase, and several studies report a delayed response of the ionospheric plasma density to solar activity changes (e.g. Jakowski et al., 1991; Astafyeva et al., 2008; Afraimovich et al., 2008; Lee et al., 2012). In most cases, TEC is reported to be delayed against the variation of the solar radiation by 1-2 days. To interpret the ionospheric delay, Jakowski et al. (1991) performed simplified theoretical studies using a one-dimensional numerical model, and found a delayed accumulation of atomic oxygen at $180 \mathrm{~km}$ height, and this delay was due to slow diffusion of 


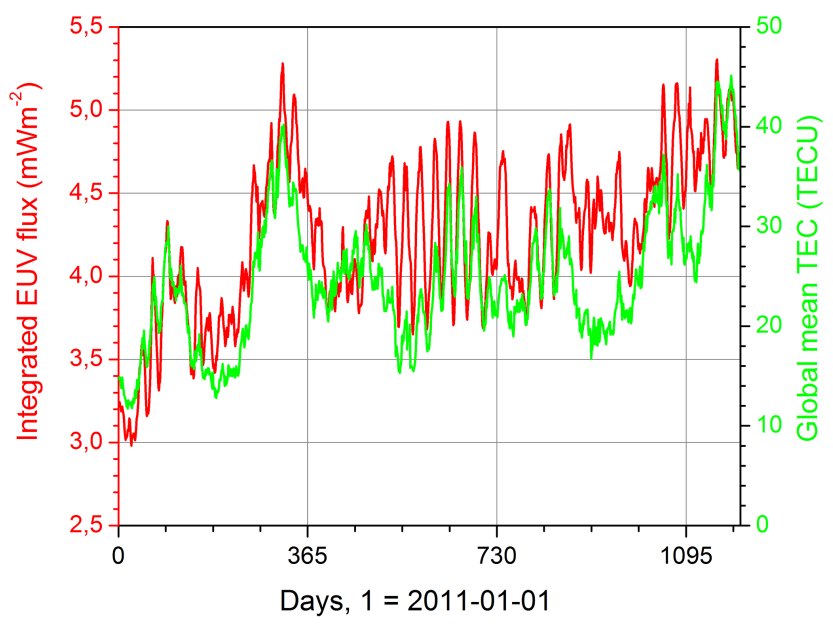

Figure 1. Time series of daily integrated EUV fluxes $(6-106 \mathrm{~nm}$, red) from SDO/EVE and daily averaged global mean TEC (green).

$\mathrm{O}$ that has been created via $\mathrm{O}_{2}$ photo-dissociation in the spectral region of the Schumann-Runge continuum from 135 to $176 \mathrm{~nm}$. Since the major F region ionization is proportional to $\mathrm{O}$, these results were consistent with the observed delayed ionospheric ionization response.

In this paper, we shall make an attempt to analyze the ionospheric delay based on datasets that represent solar EUV variability. In particular, we will investigate the time lag at different time scales between about 2 weeks and 3 months. We shall use integrated EUV-fluxes from the Extreme Ultraviolet Variability Experiment (EVE) on the Solar Dynamics Observatory (SDO), the EUV-TEC proxy based on the SDO/EVE spectra and the NRLMSISE-00 model (Picone et al., 2002). We shall use data from 2011 through early spring 2014, and analyze the correlation between EUV proxies and global TEC variability, as well as the ionospheric delay at different time scales.

\section{Data and analysis}

SDO was launched on 11 February 2010 (Pesnell et al., 2012), and data are available from 1 May 2010. EVE onboard SDO measures the solar EUV irradiance from 0.1 to $106 \mathrm{~nm}$ with a spectral resolution of $0.1 \mathrm{~nm}$, a temporal cadence of ten seconds, and an accuracy of $20 \%$ (Woods et al., 2012). The in-flight calibration for EVE includes daily measurements with redundant foil filters and on-board flatfield lamps and annual underflight calibration rocket flights. Diode-based broadband channels are also used to monitor the performance and drift of the high-resolution spectrographs.

For comparison of EUV parameters with ionospheric variability, daily mean global mean TEC values have been calculated based on International Global Navigation Satellite System Service (IGS) TEC maps (Hernandez-Pajares et al., 2009) provided by CDDIS (2015), and they will be used here to analyze the correlation between ionospheric and EUV variability. Figure 1 shows the time series of daily integrated EUV fluxes together with global mean TEC. The data represent part of the increasing phase of solar cycle 24. Naturally, at the interannual time scale, the two curves are strongly correlated. Also, correlation is strong at the time scale of the 27-day solar rotation.

We calculate the EUV-TEC proxy after Unglaub et al. (2011, 2012), which represents the vertically integrated ionization rates, and used SDO/EVE version 5 spectra (LASP, 2015) between 6 and $106 \mathrm{~nm}$ as input. The far ultraviolet (FUV) irradiance up to about $130 \mathrm{~nm}$ also contributes to ionization, but its contribution is not included in the integrated EUV band because their ionization contributions are primarily below the ionosphere $\mathrm{F}$ layer that is most important for TEC. EUV-TEC is calculated from the satellite-borne EUV measurements assuming a model atmosphere that consists of four major atmospheric constituents. Regional number densities of the background atmosphere are taken from the NRLMSISE-00 model (Picone et al., 2002). This model uses the F10.7 flux as daily input, and additionally as 81-day mean. Since the main input to EUV-TEC is the solar EUV, it is strongly correlated to the integrated EUV flux. The correlation coefficient is $r=0.997$.

The original SDO/EVE spectra integrated from 6-106 nm and the EUV-TEC proxy are subsequently used for comparison with global TEC. Because the annual cycle of global TEC and ionization is different, especially owing to the semiannual cycle in TEC, we here consider the seasonal time scale up to 3 months only. Therefore the data have been high-pass filtered using a FFT filter with a cut-off period of 3 months.

To allow comparison, the datasets were normalized by subtracting the mean and dividing by the standard deviation using the data from 16 March 2011 through 11 February 2014 (approx. Carrington rotations 2108-2146). The mean values and standard deviations are $1.438 \pm 0.078 \times$ $10^{19}$ ions $^{-2}$ for the EUV-TEC ionization rates, $4.252 \pm$ $0.238 \mathrm{mWm}^{-2}$ for SDO/EVE fluxes, and $24.9 \pm 2.1 \mathrm{TECU}$ for global TEC. The normalized time series are shown in Fig. 2. The curves are offset vertically by 2 with respect to each other. One can see that the variability, in particular the amplitude of the 27-day solar rotation, of TEC and EUV fluxes and EUV-TEC is very similar.

\section{Correlation of EUV parameters and global TEC}

Figure 3 shows scatter plots of EUV-TEC (panel a), and SDO/EVE integrated spectral flux (panel b) vs. daily mean global mean TEC. All the data have been high-pass filtered and normalized as described above. The correlation coefficient between normalized TEC and EUV-TEC is $r=0.86$ $\left(r^{2}=0.74\right)$. The correlation coefficient between normalized TEC and SDO/EVE flux is also $r=0.86$ although additional 


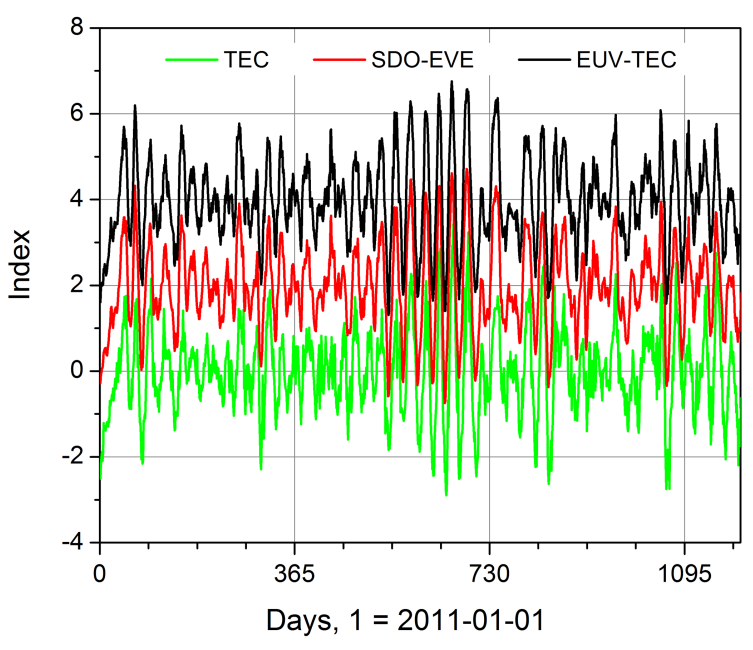

Figure 2. Time series of EUV-TEC (black), SDO/EVE integrated flux (red) and daily averaged global mean TEC (green), filtered using a high-pass filter with cut-off period of 3 months and normalized by their mean and standard deviation. The curves are offset vertically with respect to each other.

information has been added to calculate EUV-TEC. We shall discuss this below.

Figure 4 shows Morlet wavelet spectra of SDO/EVE integrated EUV fluxes (left panel) and daily mean global mean TEC (right panel). Data have been normalized and lowpass filtered as in Fig. 2. The main variation is clearly near 27 days, but there is also some power at longer time scales, although this is only intermittent.

Figure 5 shows an example of time series of SDO/EVE integrated EUV fluxes and global TEC, which have further been filtered using a Lanczos bandpass filter with 100 weights and cut-off periods of 25 and 29 days, so that the time series represents the respective variability within the 27day solar cycle. We note a delay of TEC with respect to solar variability. To systematically investigate the delay at different time scales, we now filtered the time series in different period bands, and the cut-off periods of the Lanczos filter were chosen in such a way that each period band ranges over 4 days, while the center of the period band was shifted from 4 to 88 days. For each pair of filtered time series, i.e. for each time scale (which was defined as the center of the respective period window), the cross-correlation coefficients were calculated. The results are shown as contour lines in Fig. 6; the line with a cross correlation of $r=0.8$ is highlighted. On the ordinate the time lag is given in degrees, and $360^{\circ}$ corresponds to the respective time scale on the abscissa. Dashed blue lines indicate lags of $0,1,2$, and 3 days, respectively. Positive lag values indicate that EUV variations lead TEC ones. Solid light blue dots are added that show the SDO/EVE flux time lag with maximum correlation which can, however, only be provided at an accuracy of one day. The black dots show these maxima again, but now calculated with using

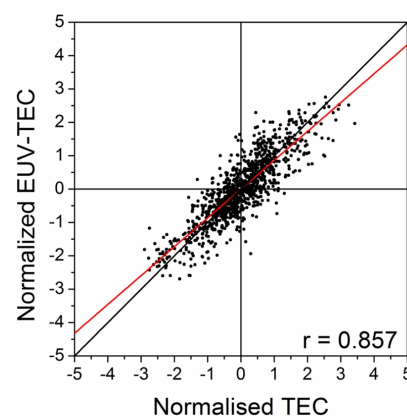

(a)

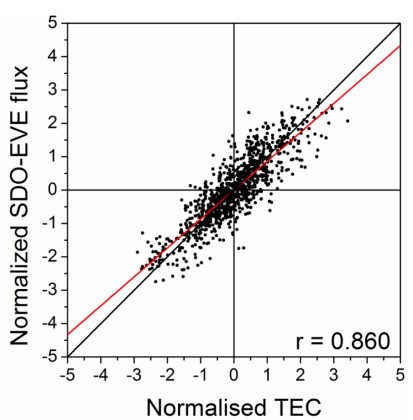

(b)
Figure 3. EUV-TEC (a), and integrated SDO/EVE flux (b) vs. global mean TEC. Data have been normalized and filtered as in Fig. 2.

EUV-TEC instead of integrated EUV fluxes. Figure 6 shows that at short time scales of few days, the correlation is weak and the shown lag values are not significant. At time scales of the solar rotation, the strongest correlation is found and solar variations lead global TEC by about one day. For slightly longer time scales, the delay increases, however, the power at the 30-40 day time scale is not strong as is shown in the wavelets in Fig. 4. For EUV-TEC, the lag increases to about 2 days for time scales around 2 months, but relative to the time scale the delay is not longer than for the 27-day cycle.

At first glance the difference in lag at time scales $>55$ days may contradict the strong correlation of integrated EUV fluxes and EUV-TEC, However, at these time scales the correlation coefficients are still large (between $r=0.8$ and $r=0.85$ ), but the amplitudes are smaller (see Fig. 4) so that this period range does not contribute very much to the total correlation. Furthermore, as can be seen in Fig. 6, the change of $r$ with lag is small near the maximum and the difference of correlation at lag 1 or 2 days is therefore small as well (both $r$ are $>99 \%$ of the maximum lag at the respective time scale). A further effect may be due to the used resolution of 1 day, and the difference of the real lags may actually be smaller than 1.

We show the same data in a different manner in Fig. 7. Now we show the ratio of the correlation coefficients and the maximum correlation for the respective time scale of the variations, which means that a horizontal contour line would denote a constant decrease of correlation with the phase of the respective variation. The light magenta region indicates the region where the ratio exceeds 0.99 . One can see that for the time scales of about 20-40 days this region is more or less constant (the fluctuations occur because we have a time resolution of 1 day only) at about $15^{\circ}$, which means that the ionospheric delay is not constant, but it is more or less a constant fraction of the time scale of the solar EUV and TEC fluctuations. For longer time scales of more than 40 days, this relative delay decreases abruptly and then again remains approximately constant. Although, owing to the temporal res- 


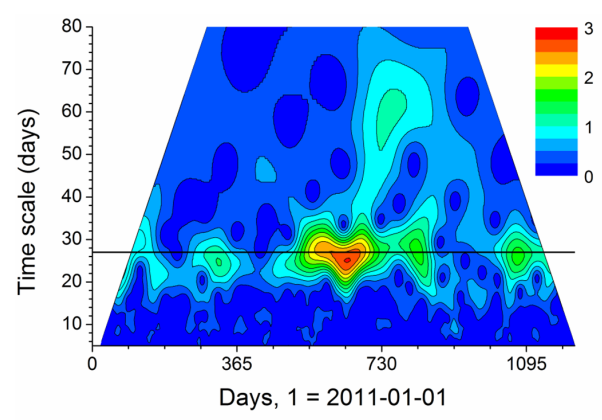

(a)

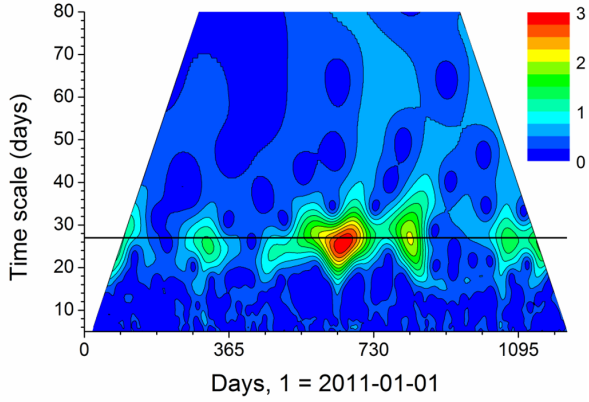

(b)

Figure 4. Morlet wavelet spectra of (a) SDO/EVE integrated EUV fluxes and (b) daily mean global mean TEC. Data have been normalized and filtered as in Fig. 2.

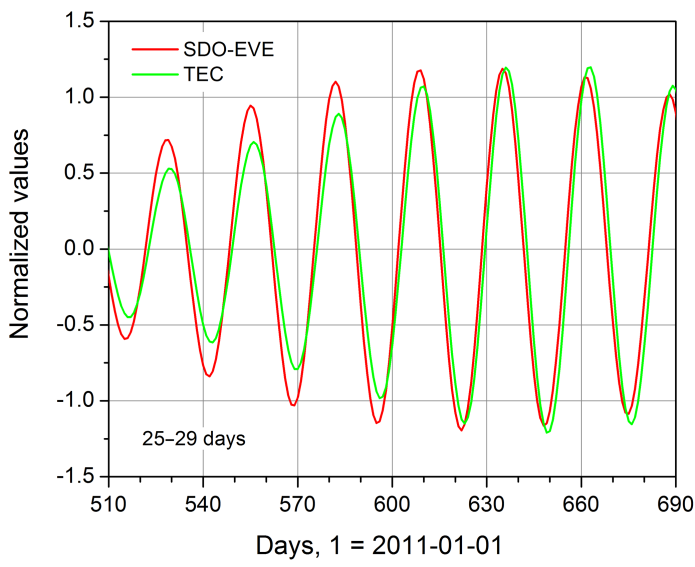

Figure 5. Example of normalized SDO/EVE integrated EUV fluxes and global mean TEC, additionally filtered in the 25-29 days period range.

olution used here, these findings cannot be considered as a proof for this behavior of the ionospheric delay, they may indicate that the processes leading to the delay at different time scales may be different.

We have made an attempt to include the ionospheric delay shown in Figs. 6 and 7 into the EUV and EUV-TEC time series. The time series had been filtered in the respective time ranges, the shifted by the delay (0-2 days), and then the filtered series had been added up again. As expected, the correlation increases, namely to $r=0.88$ for EUV-TEC and 0.89 for SDO/EVE EUV, respectively. We note that EUV-TEC describes global TEC fluctuations still a little worse than the EUV fluxes do. We also made first analyses of the EUVTEC proxy using an index based on SDO/EVE EUV fluxes integrated from 6-106 nm instead of F10.7 in NRLMSISE00. This leads to a slightly stronger correlation of EUV-TEC with TEC, but still it is not stronger than using EUV directly. Clearly, EUV-TEC is a very simple proxy. A much more sophisticated parameterization for use in general circulation models have been constructed by Solomon and Qian (2005).

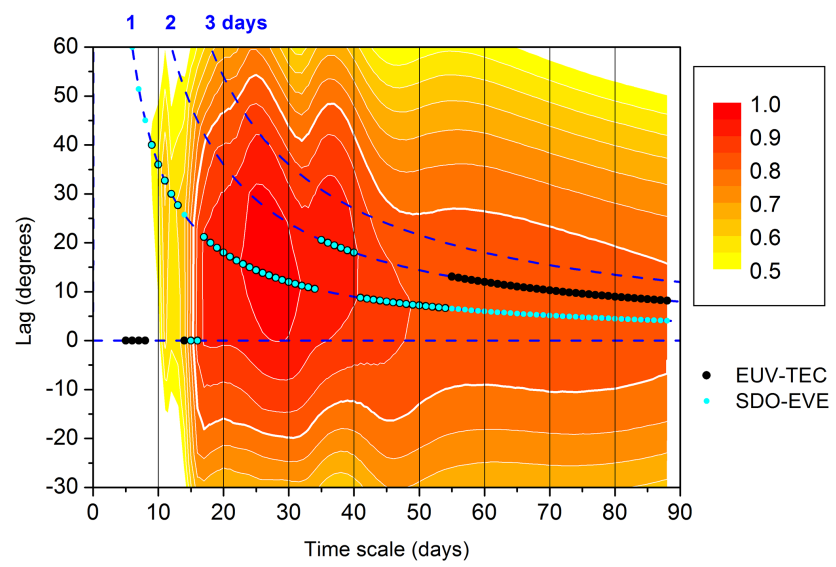

Figure 6. Cross-correlation coefficients between filtered global TEC and SDO/EVE integrated EUV fluxes. The time scale on the abscissa defines the center of the 4-day period band of the respective filter. On the ordinate the time lag is given in degrees, and $360^{\circ}$ corresponds to the respective time scale on the abscissa. Positive lag values indicate that EUV variations lead TEC ones. The dashed blue lines indicate lags of $0,1,2,3$ days as indicated on top of the figure. The light blue dots show the lag with maximum correlation at an accuracy of one day. The black dots show these maxima, but calculated using EUV-TEC instead of integrated EUV fluxes.

\section{Conclusions}

We analyzed the correlation between global mean TEC and solar EUV variability, the latter described by 2 different proxies, namely the integrated EUV flux measured by SDO/EVE, and the EUV-TEC proxy that describes primary ionization based on EUV spectra. There is an ionospheric delay at the solar rotation and longer time scale of 1-2 days, such that TEC variations lag EUV ones. There is some indication that this delay is constant when taken relative to the time scale of the EUV variations, i.e. increases slightly for variations from 20 to 40 days. For longer time scales up to 90 days, the relative delay decreases, but then remains constant again. We did not investigate time scales longer than 90 days. 


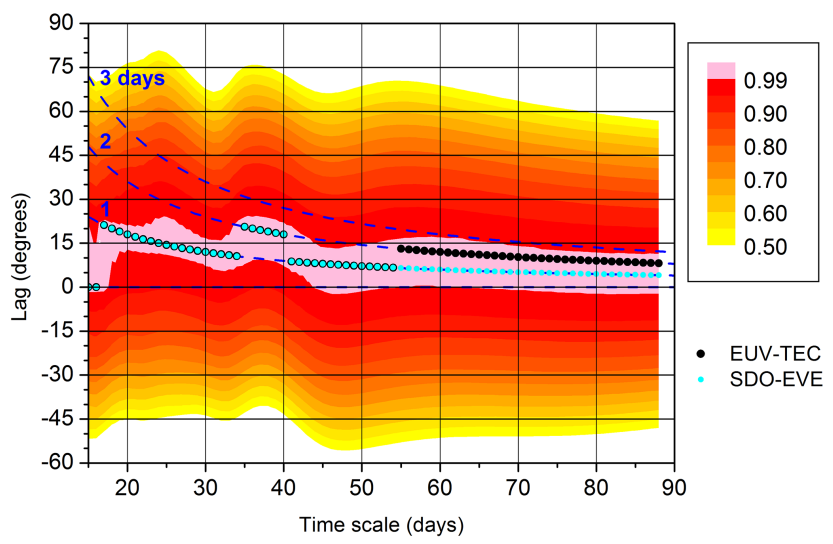

Figure 7. As in Fig. 6, but shown is the ratio of the cross-correlation coefficient and the one at the lag of maximum correlation.

It should, however, be stated here that the EUV fluxes and the ionization rates calculated by the EUV-TEC model represent only a coarse description of global TEC, and they are not well suited to describe ionization e.g. in circulation models. A parameterization of ionization and dissociation rates including photoelectron effects using solar spectral measurements or models has been presented by Solomon and Qian (2005). When used as a TEC proxy, EUV-TEC does not account for dynamics, secondary ionization, or ionization through particle precipitation. It does not take into account effects of ionospheric storms, which are a challenge for TEC forecast (Borries et al., 2015). Nevertheless, the integrated EUV flux or EUV-TEC describes TEC variations well, especially at the time scale of the solar rotation.

Obviously, the results presented here are preliminary. We used daily EUV spectra and daily and globally averaged TEC, which gives only coarse values for the ionospheric delay. Furthermore, F10.7 is observed at local noon and this may lead to a bias between F10.7 used in NRLMSISE-00 and daily TEC. TEC maps are available at higher temporal resolution, and EUV fluxes at least for some spectral bands are also available e.g. from the Solar and Heliospheric Observatory/Solar Extreme Ultraviolet Monitor (SOHO/SEM, Judge et al., 1998) or the Geostationary Operational Environmental Satellites (GOES). This provides the possibility to study ionospheric delay in higher temporal resolution and spatially resolved. However, for the calculation of the EUVTEC index spectral resolution is required, so that this would only provide a guidance for further improvements. There are still some further shortcomings of EUV-TEC. One aspect is probably the use of F10.7 in the NRLMSISE-00 atmosphere model used, so that EUV-TEC in this version is based on EUV spectra and F10.7. First preliminary results using the EUV-TEC proxy including NRLMSISE-00 based on EUV fluxes showed slightly better correlation with TEC, however, still gave slightly weaker correlation than using EUV integrated fluxes alone.

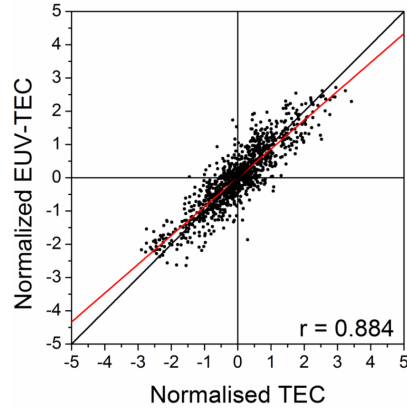

(a)

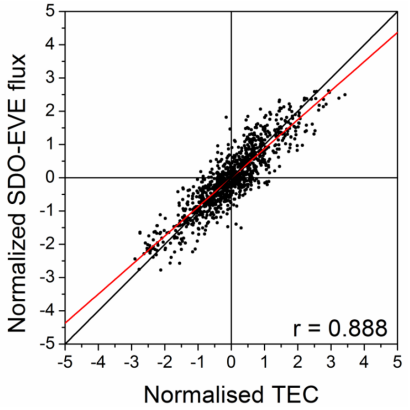

(b)
Figure 8. As in Fig. 3, but with EUV-TEC and SDO/EVE integrated fluxes shifted according to the delay as shown in Figs. 6 and 7.

Another aspect is that we completely neglect the thermosphere and its dynamics in the analysis. If the delay should be caused by dynamical processes as suggested by Jakowski et al. (1991), it is at least partly driven by FUV radiation and therefore the spectra used here are not necessarily sufficient to describe the variability. Further analyses, therefore will take this in to account, e.g. by using the MgII index instead of EUV, or in a combination.

\section{Code availability}

The EUV-TEC model can be obtained from the corresponding author on request. The code includes the NRLMSIS-00 model provided by CCMC via ftp://hanna.ccmc.gsfc.nasa. gov/pub/modelweb/atmospheric/msis/nrlmsise00/.

\section{Data availability}

IGS gridded TEC data has been provided via NASA through ftp://cddis.gsfc.nasa.gov/gps/products/ionex/. Daily F10.7 solar proxies have been provided by NGDC via http://www. ngdc.noaa.gov/stp/space-weather/solar-data/solar-features/ solar-radio/noontime-flux/penticton/penticton_observed/. The daily SDO/EVE version 5 spectra are available at LASP through http://lasp.colorado.edu/eve/data_access/ evewebdataproducts/merged/.

Acknowledgements. The original version of the EUV-TEC model has been written by C. Unglaub, Leipzig. We acknowledge support from the German Research Foundation (DFG) and Universität Leipzig within the program of Open Access Publishing.

Edited by: M. Förster

Reviewed by: two anonymous referees 


\section{References}

Afraimovich, E. L., Astafyeva, E. I., Oinats, A. V., Yasukevich, Yu. V., and Zhivetiev, I. V.: Global electron content: a new conception to track solar activity, Ann. Geophys., 26, 335-344, doi:10.5194/angeo-26-335-2008, 2008.

Astafyeva, E. I., Afraimovich, E. L., Oinats, A. V., Yasukevich, Yu. V., and Zhivetiev, I. V.: Dynamics of global electron content in 1998-2005 derived from global GPS data and IRI modeling, Adv. Space Res., 42, 763-769, 2008.

Borries, C., Berdermann, J., Jakowski, N., and Wilken, V.: Ionospheric storms - a challenge for empirical forecast of the Total Electron Content, J. Geophys Res.-Space, 120, 3175-3186, doi:10.1002/2015JA020988, 2015.

CDDIS: GNSS Atmospheric Products, available at: http://cddis.nasa.gov/Data_and_Derived_Products/GNSS/ atmospheric_products.html, last access: 19 June 2015.

Hernandez-Pajares, M., Juan, J. M., Sanz, J., Orus, R., Garcia-Rigo, A., Feltens, J., Komjathy, A., Schaer, S. C., and Krankowski, A.: The IGS VTEC maps: a reliable source of ionospheric information since 1998, J. Geod., 83, 263-275, 2009.

Jakowski, N., Fichtelmann, B., and Jungstand, A.: Solar activity control of ionospheric and thermospheric processs, J. Atmos. Terr. Phys., 53, 1125-1130, 1991.

Judge, D. L., McMullin, D. R., Ogawa, H. S., Hovestadt, D., Klecker, B., Hilchenbach, M., Möbius, E., Canfield, L. R., Vest, R. E., Watts, R., Tarrio, C., Kühne, M., and Wurz, P.: First Solar EUV Irradiances Obtained from SOHO by the Celias/Sem, Solar Phys., 177, 161-173, 1998.

LASP: Extreme ultraviolet Variability Experiment - Data, available at: http://lasp.colorado.edu/home/eve/data/data-access/, last access: 14 July 2015.

Lee, C.-K., Han, S.-C., Bilitza, D., and Seo, K.-W.: Global characteristics of the correlation and time lag between solar and ionospheric parameters in the 27-day period, J. Atmos. Sol.-Terr. Phys., 77, 219-224, doi:10.1016/j.jastp.2012.01.010, 2012.

Maruyama, T.: Solar proxies pertaining to empirical ionospheric total electron content models, J. Geophys. Res., 115, A04306, doi:10.1029/2009JA014890, 2010.

NRCC: F10.7 radio flux index, available at: http://www.ngdc.noaa. gov/stp/space-weather/solar-data/solar-features/solar-radio/ noontime-flux/, last access: 24 September 2014.
Pesnell, W. D., Thompson, B. J., and Chamberlin, P. C.: The Solar Dynamics Observatory (SDO), Solar Phys., doi:10.1007/s11207011-9841-3, 2012.

Picone, J. M., Hedin, A. E., and Drob, D. P.: NRLMSISE00 empirical model of the atmosphere: statistical comparisons and scientific issues, J. Geophys. Res., 107, 1468, doi:10.1029/2002JA009430, 2002.

Solomon, S. S. and Qian, L: Solar extreme-ultraviolet irradiance for general circulation models, J. Geophys. Res., 110, A10306, doi:10.1029/2005JA011160, 2005.

Thuillier, G. and Bruinsma, S.: The Mg II index for upper atmosphere modelling, Ann. Geophys., 19, 219-228, doi:10.5194/angeo-19-219-2001, 2001.

Tobiska, W. K.: Validating the solar EUV Proxy, E10.7, J. Geophys. Res., 106, 29969-29978, doi:10.1029/2000JA000210, 2001.

Unglaub, C., Jacobi, Ch., Schmidtke, G., Nikutowski, B., and Brunner, R.: EUV-TEC proxy to describe ionospheric variability using satellite-borne solar EUV measurements: first results, Adv. Space Res., 47, 1578-1584, doi:10.1016/j.asr.2010.12.014, 2011.

Unglaub, C., Jacobi, Ch., Schmidtke, G., Nikutowski, B., and Brunner, R.: EUV-TEC proxy to describe ionospheric variability using satellite-borne solar EUV measurements, Adv. Radio Sci., 10, 259-263, doi:10.5194/ars-10-259-2012, 2012.

Woods, T. N., Bailey, S., Eparvier, F., Lawrence, G., Lean, J., McClintock, B., Roble, R., Rottmann, G. J., Solomon, S. C., Tobiska, W. K., and White, O. R.: TIMED Solar EUV Experiment, Phys. Chem. Earth Pt. C, 25, 393-396, 2000.

Woods, T. N., Eparvier, F., Bailey, S., Chamberlin, P., Lean, J., Rottmann, G. J., Solomon, S. C., Tobiska, W. K., and Woodraska, D. L.: Solar EUV Experiment (SEE): Mission overview and first results, J. Geophys. Res., 110, A01312, doi:10.1029/2004JA010765, 2005.

Woods, T. N., Eparvier, F. G., Hock, R., Jones, A. R., Woodraska, D., Judge, D., Didkovsky, L., Lean, J., Mariska, J., Warren, H., McMullin, D., Chamberlin, P., Berthiaume, G., Bailey, S., FullerRowell, T., Sojka, J., Tobiska, W. K., and Viereck, R.: Extreme Ultraviolet Variability Experiment (EVE) on the Solar Dynamics Observatory (SDO): Overview of Science Objectives, Instrument Design, Data Products, and Model Developments, Sol. Phys., 275, 115-143, doi:10.1007/s11207-009-9487-6, 2012. 